\section{ONE COMPREHENSIVE UNIT}

The new Sirona ORTHOPHOS XG 3D

from Dental Innovations effectively combines the advantages of 3D imaging, cutting-edge panoramic 2D imaging and the option of cephalometric technology in one comprehensive unit.

The unprecedented detail and viewing angles made possible by its 3D technology with variable Field of View and High Definition Mode allow you to diagnose across a range of disciplines including implantology, endodontics and complex compactions. The ORTHOPHOS XG 3D offers a comprehensive imaging software package that allows quick viewing of both 2D and 3D images, providing the means for accurate diagnosis but with the lowest radiation dose possible. Patented easy and accurate patient positioning completes the picture of the arguably most comprehensive imaging solution in the dental market today.

Combine this with the 'CEREC meets Implant' module and the ORTHPHOS XG
3D provides new possibilities in implant treatment planning, allowing you to determine the exact position of the implant whilst taking into account both function and final restoration position.

For more details call 08700102041 or visit www.henryschein.co.uk.

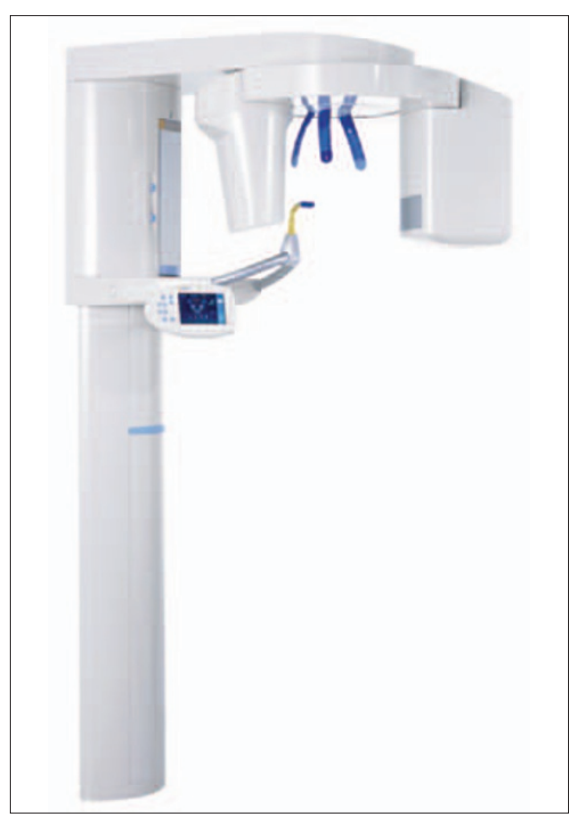

SMART MUSICAL REMINDERS FOR PATIENTS

The evidence-based information found in the Delivering better oral health toolkit is now available directly to patients in a free smartphone app called Brush DJ.

The unique app is made fun to use by playing two minutes of a song taken at random from the music stored on the user's device. Brush DJ also allows users to set reminders to:

- Clean interdentally every day

- Brush twice a day

- Use a fluoride mouthrinse if appropriate and at a different time to brushing

- Visit the dentist every 3-24 months

- Visit the hygienist if required

- Change their toothbrush/electric brush-head every three months.

The app has been launched by former BDA/Dentsply Student Clinician of the Year Ben Underwood in February and has already been downloaded in 45 countries.

The Brush

DJ app is intended as a useful motivational tool that dental professionals

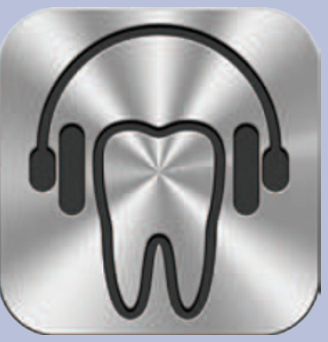
can recommend to their patients. Further information is available at www.BrushDJ.com and all feedback on how the app could be improved is very welcome. 